\title{
Simulation of supersymmetric models on the lattice without a sign problem
}

\author{
David Baumgartner* and Urs Wenger* \\ Albert Einstein Center for Fundamental Physics \\ Institute for Theoretical Physics \\ University of Bern \\ Sidlerstrasse 5 \\ CH-3012 Bern \\ Switzerland \\ E-mail: baumgarteitp.unibe.ch, wenger@itp.unibe.ch
}

\begin{abstract}
Simulations of supersymmetric models on the lattice with (spontaneously) broken supersymmetry suffer from a fermion sign problem related to the vanishing of the Witten index. We propose a novel approach which solves this problem in low dimensions by formulating the path integral on the lattice in terms of fermion loops. The formulation is based on the exact hopping expansion of the fermionic action and allows the explicit decomposition of the partition function into bosonic and fermionic contributions. We devise a simulation algorithm which separately samples the fermionic and bosonic sectors, as well as the relative probabilities between them. The latter then allows a direct calculation of the Witten index and the corresponding Goldstino mode. Finally, we present results from simulations on the lattice for the spectrum and the Witten index for $\mathscr{N}=2$ supersymmetric quantum mechanics.
\end{abstract}

The XXVIII International Symposium on Lattice Field Theory

June 14-19,2010

Villasimius, Sardinia Italy

\footnotetext{
* Speaker.
} 


\section{Introduction}

Supersymmetry (SUSY) is thought to be a crucial ingredient in the unification of the Standard Model interactions as well as in the solution of the hierarchy problem. On the other hand, we know that the low energy physics is not supersymmetric, and consequently the SUSY must be broken at low energies, either explicitly or spontaneously. Since the origin and mechanism of spontaneous SUSY breaking is a non-perturbative effect, it can not be understood in perturbation theory - instead, non-perturbative methods are required. One way to study non-perturbative effects in quantum field theories is provided by the lattice regularisation. However, the lattice discretisation comes with various problems, for example the explicit breaking of Poincare symmetry, or the absence of Leibniz' rule, and it is therefore not clear at all whether and how SUSY can be realised within the lattice regularisation.

If the lattice discretisation, for example, enjoys some exact symmetries that allow only irrelevant symmetry breaking operators, which become unimportant in the infrared regime, so-called 'accidental' symmetries may emerge from a non-symmetric lattice action and the full symmetry develops in the continuum limit. This is for example the case for (Euclidean) Poincare symmetry in lattice QCD or SUSY in $\mathscr{N}=1 \mathrm{SU}(N)$ Super-Yang-Mills theory. In the latter case, the only relevant symmetry breaking operator is the gaugino mass term which violates the $Z_{2 N}$ chiral symmetry. Therefore, a chirally invariant lattice action forbids such a term and SUSY is automatically recovered in the continuum limit. For SUSY theories involving scalar fields, however, such a way out is not available: the scalar mass term $m^{2}|\phi|^{2}$ breaks SUSY, and there is no other symmetry available to forbid that term. In principle, even in such cases, some symmetries can be obtained in the continuum by fine tuning the theory with counterterms. The restoration of chiral symmetry for Wilson fermions is one such example. For SUSY, such an approach is in general not practical, but in lower dimensions, when theories are superrenormalisable, it sometimes is [1]. Yet another approach to SUSY on the lattice is to look for an exact lattice realisation of a subalgebra of the full SUSY algebra, e.g. by combining the Poincaré and flavour symmetry group, so-called twisted SUSY (cf. [2] and references therein). This approach is applicable to systems with extended SUSY and leads to so-called $Q$-exact discretisations.

Yet another difficulty, and maybe the most severe for supersymmetry on the lattice, is the fact that supersymmetric models with broken supersymmetry inherently suffer from a fermion sign problem that hinders Monte Carlo simulations of such models on the lattice. This can easily be seen as follows. The vanishing of the Witten index

$$
W \equiv \lim _{\beta \rightarrow \infty} \operatorname{Tr}(-1)^{F} \exp (-\beta H)
$$

where $F$ is the fermion number and $H$ the Hamiltonian of the system, provides a necessary (but not sufficient) condition for spontaneous supersymmetry breaking. On the other hand, the index is equivalent to the partition function with periodic boundary conditions,

$$
W=\int_{-\infty}^{\infty} \mathscr{D} \phi \operatorname{det}[\not D(\phi)] e^{-S_{B}[\phi]}=Z_{p},
$$

and the only way for the path integral to vanish is through the fermionic determinant (or Pfaffian) being indefinite, independent of the fermion discretisation. Indeed, this has been seen in many 
studies of supersymmetric models on the lattice that allow spontaneous supersymmetry breaking, e.g. SUSY quantum mechanics with a supersymmetry breaking superpotential ${ }^{1}[3,4,5], \mathscr{N}=16$ Yang-Mills quantum mechanics [6, 7, 8, 9], $\mathscr{N}=1$ Wess-Zumino model in 2D [10], or $\mathscr{N}=(2,2)$ Super-Yang-Mills in 2D [11] (see, however, also [12, 13]).

Here we propose a novel approach based on $[14,15]$ that circumvents the fermionic sign problem by formulating the path integral on the lattice in terms of fermion loops. The formulation is based on the exact hopping expansion of the fermionic action and allows the explicit decomposition of the partition function into bosonic and fermionic contributions. Consequently, one can then devise a simulation algorithm that separately samples the fermionic and bosonic sectors, as well as the relative probabilities between them. This then allows a precise calculation of the Witten index and a direct determination of the presence or absence of a Goldstino mode. Furthermore, although this is less relevant in the present context, the approach eliminates critical slowing down and also allows simulations directly in the massless limit or at negative bare mass values [14].

\section{Fermion sign problem from spontaneous SUSY breaking}

Let us briefly elaborate further on the issue of spontaneous SUSY breaking (SSB), the vanishing of the Witten index and the connection to the fermion sign problem.

It is well known that the Witten index provides a necessary, but not sufficient, condition for SSB [16]. One has

$$
W \equiv \lim _{\beta \rightarrow \infty} \operatorname{Tr}(-1)^{F} \exp (-\beta H) \Rightarrow\left\{\begin{array}{lr}
=0 & \text { SSB may occur, } \\
\neq 0 & \text { no SSB. }
\end{array}\right.
$$

From the definition it is clear that the index counts the difference between the number of $n_{B}$ bosonic and $n_{F}$ fermionic zero energy states:

$$
W \equiv \lim _{\beta \rightarrow \infty}\left[\operatorname{Tr}_{B} \exp (-\beta H)-\operatorname{Tr}_{F} \exp (-\beta H)\right]=n_{B}-n_{F} .
$$

In a field theoretic language the index is equivalent to the partition function of the system with periodic boundary conditions imposed on both the bosonic and fermionic degrees of freedom,

$$
W=\int_{-\infty}^{\infty} \mathscr{D} \phi \operatorname{det}[\not D(\phi)] e^{-S_{B}(\phi)}=Z_{p} .
$$

Here the determinant has been obtained by integrating out the complex valued Dirac fermion fields ${ }^{2}$, while $S_{B}(\phi)$ is the action for the bosonic degrees of freedom, collectively denoted by $\phi$. It is now clear that in order to obtain a vanishing Witten index, we need both positive and negative contributions to the path integral, and this can only be achieved by the fermion determinant being indefinite. This is the source of the fermion sign problem in the context of spontaneous SUSY breaking, and we argue that such a sign problem must occur in any model aspiring to accommodate spontaneous SUSY breaking.

\footnotetext{
${ }^{1}$ Note that in the context of SUSY quantum mechanics it is misleading to speak of spontaneous or dynamical SUSY breaking; it is rather a static breaking determined by the form of the superpotential.

${ }^{2}$ In case one is dealing with real-valued Majorana fermion fields, one obtains the Pfaffian $\operatorname{Pf}[\not D(\phi)]$ instead of the determinant.
} 
It is instructive to illustrate the argument in the explicit example of SUSY quantum mechanics. The continuum action of $\mathscr{N}=2$ supersymmetric quantum mechanics reads

$$
S=\int d t \frac{1}{2}\left(\frac{d \phi(t)}{d t}\right)^{2}+\frac{1}{2} P^{\prime}(\phi(t))^{2}+\bar{\psi}(t)\left(\frac{d}{d t}+P^{\prime \prime}(\phi(t))\right) \psi(t),
$$

where the real field $\phi$ denotes the bosonic coordinate, while $\bar{\psi}$ and $\psi$ denote the two fermionic coordinates. $P(\phi)$ is the superpotential and the derivatives $P^{\prime}$ and $P^{\prime \prime}$ are taken with respect to $\phi$. The (regulated) fermion determinant with periodic boundary conditions can be calculated analytically $[17,18]$,

$$
\operatorname{det}\left[\frac{\partial_{t}+P^{\prime \prime}(\phi)}{\partial_{t}+m}\right]_{p}=\sinh \int_{0}^{T} \frac{P^{\prime \prime}(\phi)}{2} d t
$$

and by rewriting the sinh-function in terms of two exponentials, one can separate the positive and negative, or rather, the bosonic and fermionic contributions to the partition function,

$$
\operatorname{det}\left[\frac{\partial_{t}+P^{\prime \prime}(\phi)}{\partial_{t}+m}\right]_{p}=\frac{1}{2} \exp \left(+\int_{0}^{T} \frac{P^{\prime \prime}(\phi)}{2} d t\right)-\frac{1}{2} \exp \left(-\int_{0}^{T} \frac{P^{\prime \prime}(\phi)}{2} d t\right) \Longrightarrow Z_{0}-Z_{1} .
$$

As an example, consider the superpotential $P_{e}(\phi)=\frac{1}{2} m \phi^{2}+\frac{1}{4} g \phi^{4}$, which is even under the parity transformation $\phi \rightarrow \tilde{\phi}=-\phi$. In this case one finds $P_{e}^{\prime \prime}(\phi) \geq 0$ and hence $Z_{0} \neq Z_{1}$, i.e. no SUSY breaking. On the other hand, for the superpotential $P_{o}(\phi)=-\frac{\mu^{2}}{4 \lambda} \phi+\frac{1}{3} \lambda \phi^{3}$, which is odd under parity, one has $P_{o}^{\prime \prime}(\tilde{\phi})=-P_{o}^{\prime \prime}(\phi)$ and, since $S_{B}(\tilde{\phi})=S_{B}(\phi)$, one finds $Z_{0}=Z_{1}$, i.e. a vanishing Witten index and the corresponding SUSY breaking. Here, the vanishing of the Witten index is guaranteed by the fact that to each configuration $\phi$ there exists another configuration $\tilde{\phi}$ (the parity transformed one) that contributes to the path integral with the same weight, but with opposite sign stemming from the fermion determinant. Furthermore, $Z_{0}=Z_{1}$ means that (in the limit of zero temperature, i.e. $\beta \rightarrow \infty$ ) the free energies of the bosonic and fermionic vacua are equal, and that proves the existence of a massless, fermionic mode connecting the two vacua, i.e. the Goldstino mode.

Turning now to SUSY quantum mechanics on the lattice one obtains with a Wilson type discretisation (cf. next section for further details)

$$
\operatorname{det}\left[\nabla^{*}+P^{\prime \prime}(\phi)\right]_{p}=\prod_{t}\left[1+P^{\prime \prime}\left(\phi_{t}\right)\right]-1,
$$

where $t$ now denotes a discrete lattice site index and $\nabla^{*}$ is the backward derivative. Also in this case one can identify the bosonic and fermionic contributions (i.e. the first and second term of the difference in eq.(2.2)), and we will show below that this separation is always explicit in the fermion loop formulation. As a side remark, let us note that in the limit of zero lattice spacing one finds

$$
\lim _{a \rightarrow 0} \operatorname{det}\left[\nabla^{*}+P^{\prime \prime}\right] \longrightarrow \exp \left(+\int_{0}^{T} \frac{P^{\prime \prime}(\phi)}{2} d t\right) \operatorname{det}\left[\partial_{t}+P^{\prime \prime}(\phi)\right],
$$

where the exponential term can be understood as coming from radiative contributions that need to be corrected by 'fine-tuning' a corresponding counterterm [19, 18]. Reconsidering the two examples for the superpotential mentioned above, we find for $P_{e}$ with $m>0$ and $g \geq 0$ that

$$
\operatorname{det}\left[\nabla^{*}+P_{e}^{\prime \prime}\right]=\prod_{t}\left[1+m+3 g \phi_{t}^{2}\right]-1>0,
$$


while for $P_{o}$ one finds

$$
\operatorname{det}\left[\nabla^{*}+P_{o}^{\prime \prime}\right]=\prod_{t}\left[1+2 \lambda \phi_{t}\right]-1
$$

which turns out to be indefinite, even when $\lambda>0$. While this is necessary in order to enable a vanishing Witten index, it imposes a serious problem on any Monte Carlo simulation, for which positive weights are strictly required. Moreover, the sign problem is severe in the sense that towards the continuum limit (i.e. when the lattice volume goes to infinity), the fluctuations of the first summand in eq.(2.3) around 1 tend to zero, such that $W \rightarrow 0$ is exactly realised in that limit. Hence, the source of the fermionic sign problem lies in the exact cancellation of the first and the second summand in eq.(2.3), i.e. of the bosonic and fermionic contribution to the partition function, and this observation also holds more generally in higher dimensions.

In the loop formulation, to be discussed in the next section, the separation of the partition function into the various fermionic and bosonic sectors is made explicit and allows the construction of a simulation algorithm that samples these sectors separately, and more importantly also samples the relative weights between them. In this way, the loop formulation eventually provides a solution to the fermion sign problem.

\section{Loop formulation and separation of fermionic and bosonic sectors}

In this section we illustrate the loop formulation and the separation of the partition function into its fermionic and bosonic sectors by means of the $\mathscr{N}=1$ Wess-Zumino model in two dimensions and $\mathscr{N}=2$ supersymmetric quantum mechanics in one dimension.

\section{1 $\mathscr{N}=1$ Wess-Zumino model in two dimensions}

The Lagrangian of the $\mathscr{N}=1$ Wess-Zumino model in two dimensions is given by

$$
\mathscr{L}=\frac{1}{2}\left(\partial_{\mu} \phi\right)^{2}+\frac{1}{2} P^{\prime}(\phi)^{2}+\frac{1}{2} \bar{\psi}\left(\partial+P^{\prime \prime}(\phi)\right) \psi
$$

where $\psi$ is a real, two-component Majorana field, $\phi$ a real bosonic field and $P(\phi)$ an arbitrary superpotential. Integrating out the fermionic Majorana fields yields a Pfaffian which in general, as discussed above, is not positive definite.

On the lattice, one can use the exact reformulation of the fermionic Majorana degrees of freedom in terms of non-intersecting, self-avoiding loops, in order to separate the contributions of the Pfaffian to the various bosonic and fermionic sectors of the partition function. A similar exact reformulation of the bosonic degrees of freedom in terms of bonds, can also be accomplished [20]. While this is not necessary for the solution of the sign problem, it provides a convenient way to simulate also those degrees of freedom without critical slowing down, and hence we will discuss this construction below.

Employing the Wilson lattice discretisation for the fermionic part of the Lagrangian in eq.(3.1) yields

$$
\mathscr{L}_{F}=\frac{1}{2} \xi^{T} \mathscr{C}\left(\gamma_{\mu} \tilde{\nabla}_{\mu}-\frac{1}{2} \nabla_{\mu}^{*} \nabla_{\mu}+P^{\prime \prime}(\phi)\right) \xi
$$


where $\xi$ now represents the real, 2-component Grassmann field, while $\mathscr{C}=-\mathscr{C}^{T}$ is the charge conjugation matrix and $\nabla_{\mu}^{*} \nabla_{\mu}$ the Wilson term. Using the nilpotency of Grassmann elements one can expand the Boltzmann factor leading to

$$
\int \mathscr{D} \xi \prod_{x}\left(1-\frac{1}{2} M\left(\phi_{x}\right) \xi_{x}^{T} \mathscr{C} \xi_{x}\right) \prod_{x, \mu}\left(1+\xi_{x}^{T} \mathscr{C} \Gamma(\mu) \xi_{x+\hat{\mu}}\right),
$$

where $M\left(\phi_{x}\right)=2+P^{\prime \prime}\left(\phi_{x}\right), \Gamma( \pm \mu)=\frac{1}{2}\left(1 \mp \gamma_{\mu}\right)$ and $x$ denotes the discrete lattice site index. Performing now the integration over the fermion field, at each site $x$ the fields $\xi_{x}^{T} \mathscr{C}$ and $\xi_{x}$ must be exactly paired in order to give a contribution to the path integral, so one finds

$$
\int \mathscr{D} \xi \prod_{x}\left(-M\left(\phi_{x}\right) \xi_{x}^{T} \mathscr{C} \xi_{x}\right)^{m(x)} \prod_{x, \mu}\left(\xi_{x}^{T} \mathscr{C} \Gamma(\mu) \xi_{x+\hat{\mu}}\right)^{b_{\mu}(x)}
$$

where the occupation numbers $m(x)=0,1$ for the monomer terms and $b_{\mu}(x)=0,1$ for the fermionic bonds (or dimers), satisfy the constraint

$$
m(x)+\frac{1}{2} \sum_{\mu}\left(b_{\mu}(x)+b_{\mu}(x-\hat{\mu})\right)=1 \quad \forall x .
$$

This constraint is equivalent to the fact that only closed, self-avoiding paths survive the Grassmann integration. When integrating out the fermion fields, the projectors $\Gamma(\mu)$ eventually yield a weight $\omega$, which only depends on the geometric structure of the specific constrained path (CP) configuration $\ell \in \mathscr{L}$. In particular, one has

$$
|\omega(\ell)|=2^{-n_{c} / 2},
$$

where $n_{c}$ denotes the number of corners in the loop configuration, while the sign depends on the topology of the loop configuration and will be discussed below.

As mentioned above, the bosonic fields can be treated analogously [20]. On the lattice, the kinetic term $\left(\partial_{\mu} \phi\right)^{2}$ yields $\phi_{x} \phi_{x-\hat{\mu}}$, and expanding this hopping term to all orders gives

$$
\int \mathscr{D} \phi \prod_{x, \mu} \sum_{n_{\mu}(x)} \frac{1}{n_{\mu}(x) !}\left(\phi_{x} \phi_{x-\hat{\mu}}\right)^{n_{\mu}(x)} \prod_{x} \exp \left(-\frac{1}{2} V\left(\phi_{x}\right)\right) M\left(\phi_{x}\right)^{m(x)}
$$

with bosonic bond occupation numbers $n_{\mu}(x)=0,1,2, \ldots$ and $V\left(\phi_{x}\right)=4+P^{\prime}\left(\phi_{x}\right)^{2}$. In contrast to the fermionic case, the exact reformulation requires one to include an infinite number of terms in the hopping expansion, and hence occupation numbers up to infinity, instead of just 0 and 1 as for the fermionic bonds. Integrating out the bosonic fields $\phi_{x}$ yields the site weights

$$
Q\left(n_{\mu}(x), m(x)\right)=\int d \phi_{x} \exp \left(-\frac{1}{2} V\left(\phi_{x}\right)\right) \phi_{x}^{N(x)} M\left(\phi_{x}\right)^{m(x)},
$$

where $N(x)=\sum_{\mu}\left(n_{\mu}(x)+n_{\mu}(x-\hat{\mu})\right)$ counts the number of bosonic bonds attached to a given site, while $M\left(\phi_{x}\right)^{m(x)}$ may contribute additional powers of $\phi_{x}$. So the bosonic contribution to the weight of a given configuration factorises into a product of local weights,

$$
W\left(n_{\mu}(x), m(x)\right)=\prod_{x, \mu} \frac{1}{n_{\mu}(x) !} \prod_{x} Q\left(n_{\mu}(x), m(x)\right) .
$$


In summary, the fermionic and bosonic degrees of freedom in our original partition function are now expressed in terms of fermionic monomers and dimers and bosonic bonds, and the integration over the fields has been replaced by a constrained sum over all allowed monomer-dimer-bond configurations, yielding

$$
Z=\sum_{\{\ell\} \in \mathscr{L}} \sum_{\left\{n_{\mu}\right\} \in C P}\left|W\left(n_{\mu}(x), m(x)\right) \cdot \omega(\ell)\right|
$$

In particular, the partition function for the fermionic degrees of freedom is represented by a sum over all non-oriented, self-avoiding fermion loops

$$
Z_{\mathscr{L}}=\sum_{\{\ell\} \in \mathscr{L}}\left|W\left(n_{\mu}(x), m(x)\right) \cdot \omega(\ell)\right|, \quad \mathscr{L} \in \mathscr{L}_{00} \cup \mathscr{L}_{10} \cup \mathscr{L}_{01} \cup \mathscr{L}_{11}
$$

where $\ell$ represents a fermion loop configuration in one of the four topological classes $\mathscr{L}_{l_{1}, l_{2}}$, with $l_{1}, l_{2}=0,1$ denoting the total number of loop windings (modulo 2) along the first and second direction, respectively, on the periodic lattice torus. $\omega$ denotes the weight of the specific loop configuration and depends on the geometry of the loop configuration, as discussed above. The sign of the weight is solely determined by the topological class and the fermionic boundary conditions $\varepsilon_{\mu}$, where $\varepsilon_{\mu}=0,1$ stands for periodic and anti-periodic boundary conditions, respectively. Configurations in $\mathscr{L}_{00}$ have positive weights independent of the boundary conditions, while the sign of the weights for configurations in $\mathscr{L}_{01}, \mathscr{L}_{10}$ and $\mathscr{L}_{11}$ is given by $(-1)^{l_{\mu} \cdot \varepsilon_{\mu}+1}$. So the partition function $Z_{\mathscr{L}}$ in eq.(3.4), where all sectors contribute positively, represents a system with unspecified fermionic boundary conditions [21], while a partition function with fermionic b.c. periodic in the spatial and anti-periodic in the temporal direction, respectively, is described by the combination

$$
Z_{p a}=Z_{\mathscr{L}_{00}}-Z_{\mathscr{L}_{10}}+Z_{\mathscr{L}_{01}}+Z_{\mathscr{L}_{11}}
$$

This combination represents the system at finite temperature. Analogously, the partition function with fermionic b.c. periodic in all directions - the Witten index - is given by

$$
Z_{p p}=Z_{\mathscr{L}_{00}}-Z_{\mathscr{L}_{10}}-Z_{\mathscr{L}_{01}}-Z_{\mathscr{L}_{11}}
$$

The interpretation of the Witten index in terms of the partition functions $Z_{\mathscr{L}_{i j}}$ is straightforward. Any fermion loop winding non-trivially around the lattice carries fermion number $F=1$, hence configurations with an odd number of windings, i.e. configurations in $Z_{\mathscr{L}_{10}}, Z_{\mathscr{L}_{01}}$ and $Z_{\mathscr{L}_{11}}$, also carry fermion number $F=1$, while configurations with no, or an even number of windings, i.e. in $Z_{\mathscr{L}_{00}}$, have $F=0$. The partition function $Z_{\mathscr{L}_{00}}$ may therefore be interpreted as representing the bosonic vacuum, while the combination $Z_{\mathscr{L}_{10}}+Z_{\mathscr{L}_{01}}+Z_{\mathscr{L}_{11}}$ corresponds to the fermionic vacuum. Consequently, the latter contributes to the Witten index $W \equiv Z_{p p}$ with opposite sign relative to the bosonic vacuum. Since each of the four partition functions is positive, vanishing of the Witten index implies $Z_{\mathscr{L}_{00}}=Z_{\mathscr{L}_{10}}+Z_{\mathscr{L}_{01}}+Z_{\mathscr{L}_{11}}$.

\section{2 $\mathscr{N}=2$ supersymmetric quantum mechanics}

The loop formulation for the $\mathscr{N}=2$ supersymmetric quantum mechanics on the lattice is obtained in a completely analogous manner. Using again the Wilson lattice discretisation for the 
fermionic part, the continuum action in eq.(2.1) reads

$$
S_{L}=\sum_{x} \frac{1}{2}\left(P^{\prime}\left(\phi_{x}\right)^{2}+2 \phi_{x}^{2}\right)-\phi_{x} \phi_{x-1}+\left(1+P^{\prime \prime}\left(\phi_{x}\right)\right) \bar{\psi}_{x} \psi_{x}-\bar{\psi}_{x} \psi_{x-1},
$$

where $x$ now denotes the one-dimensional, discrete lattice site index. Note that in one dimension the fermionic lattice derivative, including the contribution from the Wilson term with Wilson parameter $r=1$, becomes a simple, directed hop $\bar{\psi}_{x} \psi_{x-1}$ which, in the loop formulation, can be described by the (directed) bond occupation number $b(x)=0,1$. Integrating out the fermionic degrees of freedom yields a constraint for the fermion monomer and bond occupation numbers, analogous to eq.(3.2), namely

$$
m(x)+\frac{1}{2}(b(x)+b(x-1))=1 \quad \forall x .
$$

So for $\mathscr{N}=2$ supersymmetric quantum mechanics the loop formulation becomes particularly simple: there are just two different loop configurations, namely a bosonic one, where $m(x)=1, b(x)=0$ for all $x$, and a fermionic one where $m(x)=0, b(x)=1$ for all $x$. Since the latter corresponds to a closed fermion loop, it will pick up a minus sign from the Grassmann integration, relative to the bosonic contribution.

On top of the two fermion loop configurations one may treat the bosonic fields in the same way as before and employ a hopping expansion to all orders. After rearranging the bosonic fields the integration can eventually be performed separately at each site and one ends up with the weight

$$
W(n(x), m(x))=\prod_{x} \frac{1}{n(x) !} \int d \phi_{x} \phi_{x}^{n(x)+n(x-1)} \mathrm{e}^{-\frac{1}{2} V\left(\phi_{x}\right)}\left(1+P^{\prime \prime}\left(\phi_{x}\right)\right)^{m(x)}
$$

for a given bosonic and fermionic bond configuration, with $V\left(\phi_{x}\right)=2+P^{\prime}(\phi)^{2}$. In terms of these weights the partition function can now be written as

$$
Z_{\mathscr{L}}=\int \mathscr{D} \phi \mathscr{D} \bar{\psi} \mathscr{D} \psi \mathrm{e}^{-S_{L}}=\sum_{\{\ell\} \in \mathscr{L}} \sum_{\{n\} \in \mathrm{CP}}|W(n(x), m(x))|, \quad \mathscr{L} \in \mathscr{L}_{0} \cup \mathscr{L}_{1},
$$

where the second sum is over all allowed bosonic bond configurations $\{n\} \in \mathrm{CP}$, and $\ell$ represents one of the two fermion loop configurations in the topological classes $\mathscr{L}_{0}$ or $\mathscr{L}_{1}$, respectively. As before, the sign of the weight is solely determined by the topological class and the fermionic boundary condition. If $l=0,1$ denotes the fermion loop winding number and, as before, $\varepsilon=0,1$ the periodic and anti-periodic fermionic boundary condition, respectively, the sign is given by $(-1)^{l \cdot(\varepsilon+1)}$.

Choosing anti-periodic fermionic boundary conditions $\varepsilon=1$ we find

$$
Z_{a}=Z_{\mathscr{L}_{0}}+Z_{\mathscr{L}_{1}}
$$

which is simply the partition function for the system at finite temperature, while choosing periodic fermionic boundary conditions $\varepsilon=0$ yields

$$
Z_{p}=Z_{\mathscr{L}_{0}}-Z_{\mathscr{L}_{1}}
$$

representing the Witten index. Here, the interpretation is particularly intuitive: the two fermion loop configurations simply represent the bosonic and fermionic vacuum, while $Z_{\mathscr{L}_{0}}$ and $Z_{\mathscr{L}_{1}}$ represent the bosonic and fermionic partition function in the corresponding sectors. The Witten index 
vanishes whenever $Z_{\mathscr{L}_{0}}=Z_{\mathscr{L}_{1}}$, i.e. when the contributions from the bosonic and fermionic sectors cancel. In this case, the free energy of the bosonic and fermionic vacuum is equal, and this is equivalent to saying that there exists a gapless, fermionic excitation which oscillates between the two vacua, i.e. the Goldstino mode.

\section{Solution of the fermion sign problem}

In this section we briefly describe the simulation algorithm and explain how it eventually solves the fermion sign problem. The loop system can most efficiently be simulated by enlarging the configuration space by open strings. Following [20] the bosonic bonds are updated by inserting two bosonic sources, which sample directly the bosonic 2-point correlation function. Similarly, the fermion bonds are most efficiently updated by simulating a fermionic string [14] that samples the configuration space of the fermionic 2-point correlation function, instead of the standard configuration space of loops.

For the $\mathscr{N}=1$ Wess-Zumino model in two dimensions, where the fermionic degrees of freedom are Majorana, the open fermionic string is non-oriented and corresponds to the insertion of a Majorana fermion pair $\left\{\xi_{x}^{T} \mathscr{C}, \xi_{y}\right\}$ at position $x$ and $y$, while for $\mathscr{N}=2$ supersymmetric quantum mechanics, where the fermionic degrees of freedom are Dirac, the string is oriented and corresponds to the insertion of a Dirac fermion pair $\left\{\bar{\psi}_{x}, \psi_{y}\right\}$.

The algorithm proceeds by locally updating the endpoints of the open fermionic string using a simple Metropolis or heat bath step according to the weights of the corresponding 2-point function (cf. [14] for details). When one end is shifted from, say, $x$ to one of its neighbouring sites $y$, a fermionic dimer on the corresponding bond is destroyed or created depending on whether the bond is occupied or not. Contact with the partition functions $Z_{\mathscr{L}_{i j}}$ and $Z_{\mathscr{L}_{i}}$, respectively, is made each time the open string closes. This then provides the proper normalisation for the expectation value of the 2-point function.

The solution of the fermion sign problem discussed above relies on the correct determination of the relative weights between the bosonic and fermionic sectors, and the simulation algorithm described in [14] achieves this in a most efficient way. The open fermionic string tunnels between loop configurations in the various topological homotopy classes $\mathscr{L}_{00}, \mathscr{L}_{10}, \mathscr{L}_{01}, \mathscr{L}_{11}$ in two, and $\mathscr{L}_{0}, \mathscr{L}_{1}$ in one dimension, thereby determining the relative weights between the partition functions $Z_{\mathscr{L}_{00}}, Z_{\mathscr{L}_{10}}, Z_{\mathscr{L}_{01}}, Z_{\mathscr{L}_{11}}$, or $Z_{\mathscr{L}_{0}}, Z_{\mathscr{L}_{1}}$, respectively. From the relative weights, the Witten index (or any other partition function of interest) can be reconstructed a posteriori.

Let us emphasise that the open string algorithm and the corresponding absence of critical slowing down at the critical point as reported in [14] is crucial for the solution of the sign problem. Since the algorithm updates the configurations according to the fermionic 2-point correlation function, they are updated equally well on all length scales up to a scale of the order of the largest fermionic correlation length. This is in fact the reason why critical slowing down is essentially absent even at a critical point when the correlation length becomes infinite. Now, in order to have $W=0$ in the continuum $(L \rightarrow \infty)$ the Goldstino mode has to become massless. Since the algorithm ensures an efficient update of that mode, the tunneling between the bosonic and fermionic vacua is guaranteed and the Witten index indeed vanishes in practice. 

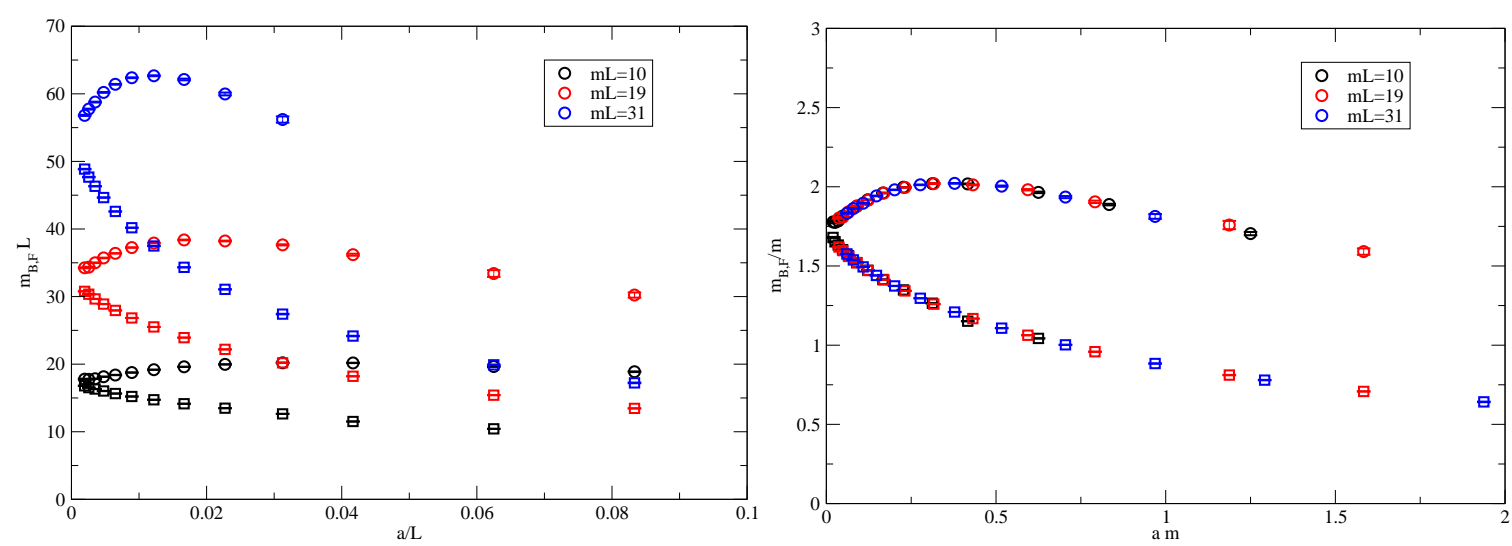

Figure 1: Spectrum for the perturbatively improved standard discretisation at $g / m^{2}=1.0$. In the left plot the excitation energies $m_{B}$ (circles) and $m_{F}$ (squares) and the lattice spacing are expressed in units of the lattice extent $L$, while in the right plot they are expressed in units of the bare mass parameter $m$.

\section{Results for $\mathscr{N}=2$ supersymmetric quantum mechanics}

We are now ready to present some results for the spectrum and the Witten index for the case of $\mathscr{N}=2$ supersymmetric quantum mechanics.

\subsection{Spectrum for the perturbatively improved standard discretisation}

Here we present our results for the spectrum using the standard discretisation including a counterterm. As discussed in [19], for the standard discretisation described above, the correct continuum limit is spoiled by radiative corrections ${ }^{3}$. This can be corrected by adding a counterterm of the form $\frac{1}{2} \sum_{x} P^{\prime}\left(\phi_{x}\right)$ [19]. By doing so, one ensures that all observables reach the correct continuum limit and that the full supersymmetry is eventually restored.

As an example we consider a superpotential with unbroken supersymmetry, i.e. $P_{e}(\phi)=$ $\frac{1}{2} m \phi^{2}+\frac{1}{4} g \phi^{4}$, and choose the coupling $g / m^{2}=1.0$. The results are presented in figure 1 where we show the lowest lying excitation energies for the boson (circles) and the fermion (squares) as a function of the lattice spacing $a$ for various values of fixed $m L$. In the left plot, the quantities are expressed in units of the lattice extent $L$, while in the right plot, they are expressed in units of the bare mass parameter $m$ in order to illustrate the common scaling behaviour. The leading lattice artifacts turn out to be $\mathscr{O}(a)$ for both the fermion and boson masses. At finite lattice spacing the supersymmetry is explicitly broken by the discretisation, and hence the boson and fermion masses are not degenerate. In the continuum limit, however, the supersymmetry is restored and the masses become degenerate.

\subsection{Spectrum for the $Q$-exact discretisation}

As briefly discussed in the introduction, for models with extended supersymmetry it is sometimes possible to preserve some of the supersymmetries exactly at finite lattice spacing [22]. The so-called $Q$-exact discretisations preserve a suitable sub-algebra of the full supersymmetry algebra,

\footnotetext{
${ }^{3}$ Note, however, that in one dimension these corrections are finite.
} 

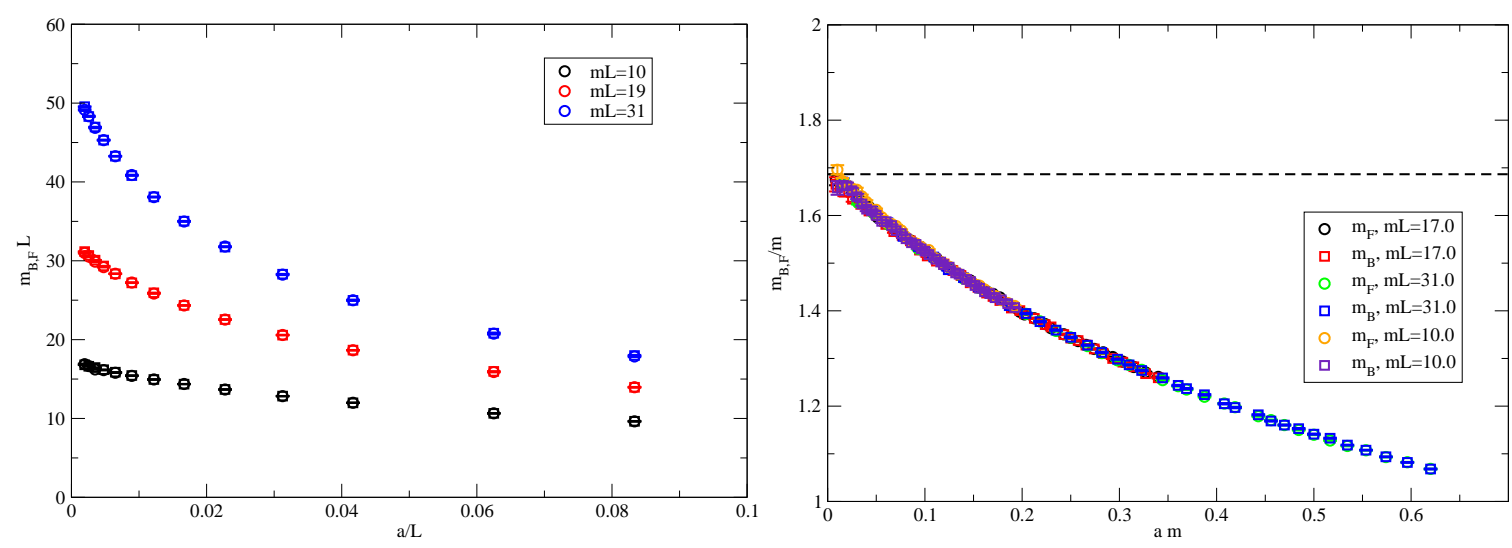

Figure 2: Spectrum for the $Q$-exact discretisation at $g / m^{2}=1.0$. In the left plot the excitation energies $m_{B}$ (circles) and $m_{F}$ (squares) and the lattice spacing are expressed in units of the lattice extent $L$, while in the right plot they are expressed in units of the bare mass parameter $m$. The dashed line denotes the exact value obtained with Numerov's method.

i.e. a linear combination of the available supersymmetries. In the context of $\mathscr{N}=2$ supersymmetric quantum mechanics the $Q$-exact action is obtained from the standard action by adding, e.g., the term $\sum_{x} P^{\prime}\left(\phi_{x}\right) \nabla^{*} \phi_{x}$, but other forms are also possible [23, 18]. Since the term contains a derivative, there are additional hopping terms that need to be considered in the hopping expansion [24].

In the following we concentrate again on the superpotential $P_{e}$ with unbroken supersymmetry. Using the $Q$-exact discretisation one expects degenerate fermion and boson masses even at finite lattice spacing [22] and this is beautifully confirmed by our results at $\mathrm{g} / \mathrm{m}^{2}=1.0$ presented in figure 2. Note that the leading lattice artifacts are again $\mathscr{O}(a)$ for both the fermion and boson masses.

Finally, in the right plot we show the results of a high precision simulation that serves the purpose of checking the correctness of the new simulation algorithm, as well as our procedures for the extraction of the fermion and boson masses. Indeed, we can confirm the mass degeneracy to a precision better than a few per mill at all lattice spacings, and the continuum value of the mass gap agrees with the exact result in the continuum obtained with Numerov's method (dashed line) also within a few per mill. Due to the loop formulation and the efficiency of the new simulation algorithm, these results can be obtained with a very modest computational effort.

\subsection{Witten index}

Let us turn to the Witten index $W \propto Z_{p} / Z_{a}$, i.e. the partition function with periodic boundary conditions $Z_{p}$, normalised to the finite temperature partition function $Z_{a}$. We start with the superpotential $P_{e}(\phi)=\frac{1}{2} m \phi^{2}+\frac{1}{4} g \phi^{4}$ for which supersymmetry is unbroken and $W \neq 0$. The results are presented in figure 3 , where we show $Z_{p} / Z_{a}$ in the left plot as a function of the bare mass parameter am for the coupling $\mathrm{g} / \mathrm{m}^{2}=1.0$ using the $Q$-exact discretisation. The continuum limit is reached as $a m \rightarrow 0$, and we indeed find that $Z_{p} / Z_{a} \rightarrow 1$, i.e. $W \neq 0$ in that limit on a sufficiently large lattice. The fact that at fixed lattice extent $L$ the partition function ratio goes to zero with $a m \rightarrow 0$ can be interpreted as a 'finite size', or rather finite temperature effect, since the temperature $T$ is inversely proportional to the extent of the lattice $L$. So if we plot the data as a function of $m / T=m L, m L=0$ corresponds to infinitely high temperature, while the limit $m L \rightarrow \infty$ corresponds to $T \rightarrow 0$. This is 

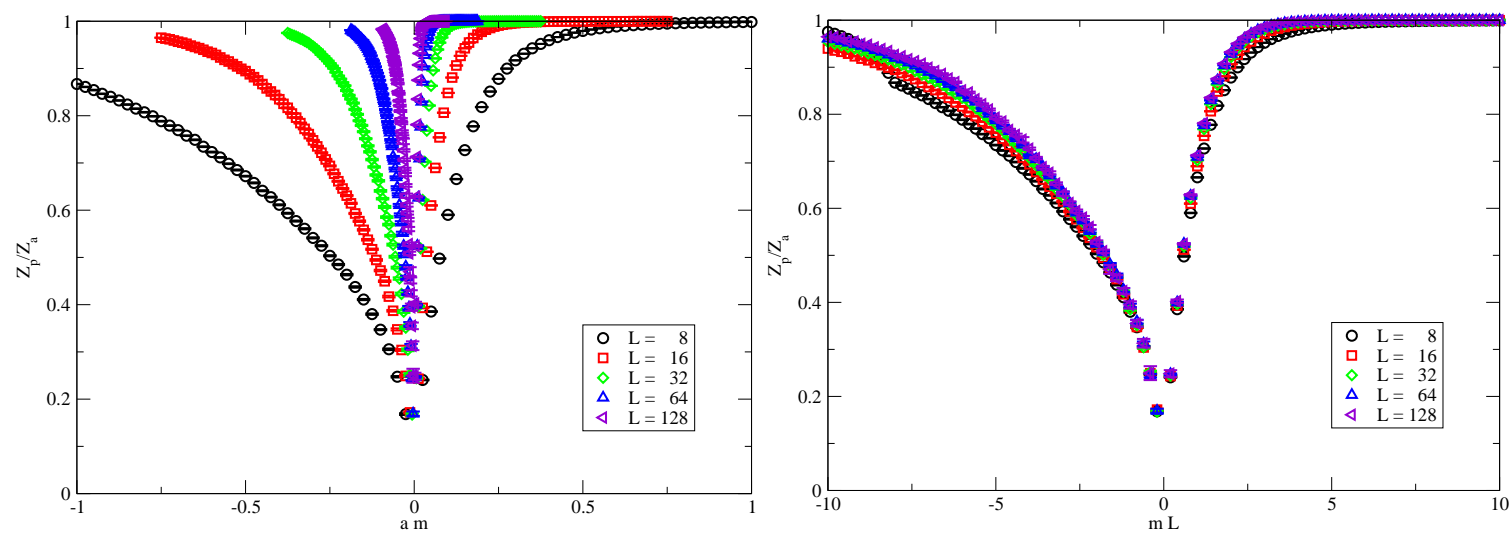

Figure 3: Simulation results for the Witten index $W \propto Z_{p} / Z_{a}$ as a function of the bare mass parameter am (left plot), and as a function of the inverse temperature $m L$ (right plot), for a system with unbroken supersymmetry using the $Q$-exact discretisation at $g / \mathrm{m}^{2}=1.0$.
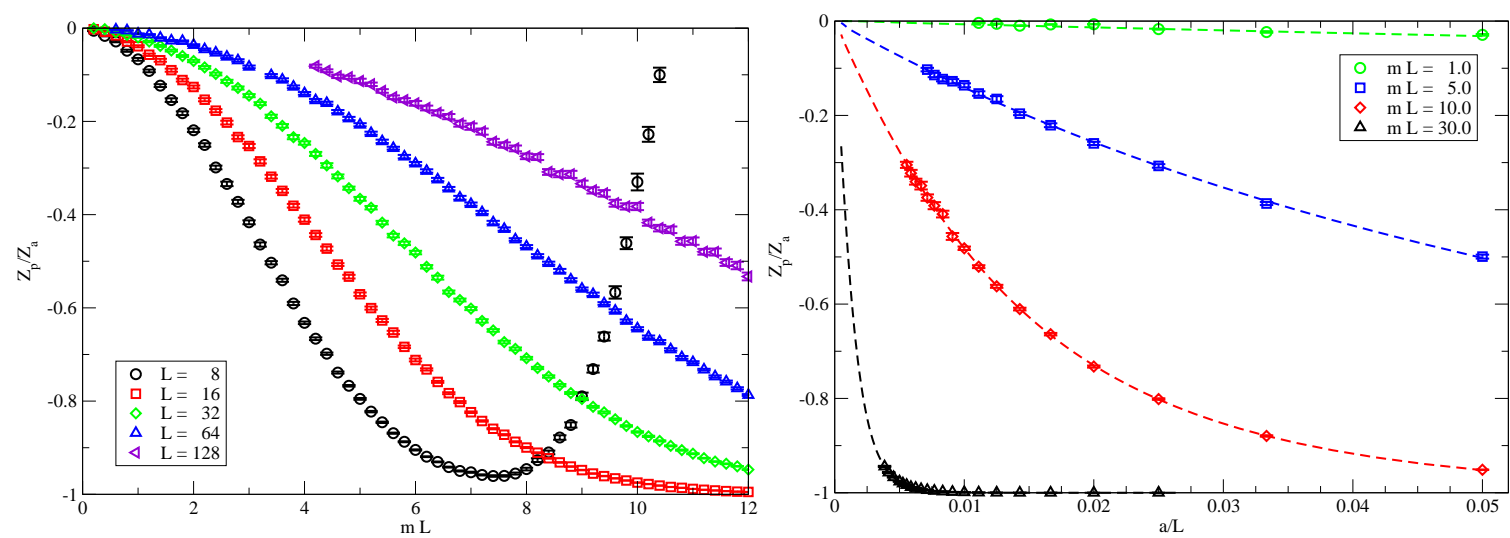

Figure 4: Simulation results for the Witten index $W \propto Z_{p} / Z_{a}$ as a function of the inverse temperature $m L$ (left plot), and as a function of the lattice spacing $a / L$ at fixed temperature (right plot), for a system with broken supersymmetry using the standard, perturbatively improved discretisation at $\lambda^{2} / \mathrm{m}^{3}=1.0$.

illustrated in the right plot of figure 3 , from where we find $Z_{p} / Z_{a} \rightarrow 1$ in the continuum limit for temperatures $m L \gtrsim 5$. The data shows a rather good scaling behaviour towards the continuum limit $\left(L \rightarrow \infty\right.$ at fixed $m L$ ). In the continuum, the value $Z_{p} / Z_{a}=1$ is approached exponentially fast with $m L$, i.e. towards zero temperature $m L \rightarrow \infty$.

Note that with the formulation and algorithm presented here, it is also possible to simulate at negative bare mass $m<0$. In this case, we still find $Z_{p} / Z_{a} \rightarrow 1$ in the continuum limit towards zero temperature, albeit at a slower rate.

When repeating this exercise for the superpotential $P_{o}(\phi)=-\frac{m^{2}}{4 \lambda} \phi+\frac{1}{3} \lambda \phi^{3}$, for which supersymmetry is broken, we should expect a vanishing Witten index. Our results for this case are presented in figure 4, where we show the Witten index $W \propto Z_{p} / Z_{a}$ as a function of $m L$ in the left plot, and as a function of the lattice spacing $a / L$ at fixed values of $m L$ in the right plot using the standard, perturbatively improved discretisation at $\lambda^{2} / \mathrm{m}^{3}=1$.0. In this case we find that while the Witten index $W \propto Z_{p} / Z_{a}$ approaches zero at 'infinite temperature' $m L \rightarrow 0$ as in the unbroken case, it also does so at any value of $m L$ in the continuum limit, even though for large values of $m L$ the 
scaling towards the continuum limit is reached only at very fine lattice spacings. In fact, for large $m L$ the approach $W \rightarrow 0$ is exponentially slow in the lattice spacing. This is exemplified in the right plot of figure 4, where the dashed lines are the results of an analytic calculation which will be reported elsewhere [24]. The absence of critical slowing down for our update algorithm guarantees that reliable results can be obtained despite the exponentially slow approach to the continuum.

\section{Summary and outlook}

We have discussed the occurrence of a fermion sign problem in the context of spontaneous supersymmetry breaking on the lattice and its relevance for the vanishing of the Witten index, regulated as a path integral on the lattice. We then argued that with the help of the fermion loop expansion one can achieve an explicit separation of the bosonic and fermionic contributions to the path integral in such a way that the source of the sign problem, namely the cancellation between the bosonic and fermionic contributions to the partition function with periodic boundary conditions, is isolated. The solution of the fermion sign problem is then achieved by devising an algorithm which separately samples the bosonic and fermionic contributions to the partition functions and, in addition, also samples the relative weights between them, essentially without any critical slowing down. In such a way one is able to calculate the Witten index on the lattice without suffering from the fermion sign problem even when the index vanishes. The absence of critical slowing down is essentially due to the fact that the algorithm directly samples the massless Goldstino mode which mediates the tunnelings between the bosonic and fermionic vacua.

As examples we described in some detail the exact reformulation of the lattice path integral in terms of fermionic bonds and monomers, and bosonic bonds for the $\mathscr{N}=1$ supersymmetric Wess-Zumino model in 2 dimensions and $\mathscr{N}=2$ supersymmetric quantum mechanics. For the latter we presented some results from lattice simulations. For a superpotential with unbroken supersymmetry, we calculate the energy gap of the lowest bosonic and fermionic excitation using both the standard discretisation with a fine-tuned counterterm, as well as a $Q$-exact discretisation which preserves a linear combination of the two supersymmetries. In the latter case the boson and fermion spectra are degenerate even at finite lattice spacing, while in the former case they become degenerate only in the continuum limit. Finally, we also present lattice calculations of the Witten index for broken and unbroken supersymmetry using the standard discretisation with a counterterm. For both cases we are able to reproduce the correct Witten index in the continuum limit. For broken supersymmetry the approach to the continuum limit is exponentially slow in the lattice spacing. Using the loop formulation and the fermion worm algorithm [14] the exponentially slow approach as well as the sign problem is no obstacle in practice.

Obviously, the approach presented here is particularly interesting for the $\mathscr{N}=1$ supersymmetric Wess-Zumino model in 2 dimensions, where so far simulations on the lattice have suffered from the fermion sign problem [10]. Work in this direction is in progress.

\section{References}

[1] M. F. L. Golterman and D. N. Petcher, Nucl. Phys. B319 (1989) 307-341.

[2] S. Catterall, PoS LATTICE2010 (2010) 002, [arXiv: 1010 . 6224]. 
[3] I. Kanamori, H. Suzuki, and F. Sugino, Phys. Rev. D77 (2008) 091502, [arXiv: 0711.2099 ].

[4] I. Kanamori, Nucl. Phys. B841 (2010) 426-447, [arXiv:1006.2468].

[5] I. Kanamori, PoS LATTICE2010 (2010) 254, [arXiv: 1011.094 0].

[6] S. Catterall and T. Wiseman, JHEP 12 (2007) 104, [arXiv: 0706.3518$].$

[7] K. N. Anagnostopoulos, M. Hanada, J. Nishimura, and S. Takeuchi, Phys. Rev. Lett. 100 (2008) 021601, [arXiv:0707.4454].

[8] M. Hanada, A. Miwa, J. Nishimura, and S. Takeuchi, Phys. Rev. Lett. 102 (2009) 181602, [arXiv:0811.2081].

[9] M. Hanada, arXiv:1011.1284.

[10] S. Catterall and S. Karamov, Phys. Rev. D68 (2003) 014503, [hep-lat/ 0305002 ].

[11] J. Giedt, Nucl. Phys. B668 (2003) 138-150, [hep-lat/ 0304006 ].

[12] I. Kanamori and H. Suzuki, Nucl. Phys. B811 (2009) 420-437, [arXiv: 0809.2856 ].

[13] M. Hanada and I. Kanamori, JHEP 01 (2011) 058, [arXiv: 1010.2948 ].

[14] U. Wenger, Phys. Rev. D80 (2009) 071503, [arXiv: 0812 . 3565].

[15] U. Wenger, PoS LAT2009 (2009) 022, [arXiv: 0911.4099 ].

[16] E. Witten, Nucl. Phys. B202 (1982) 253.

[17] F. Cooper, A. Khare, and U. Sukhatme, Phys. Rept. 251 (1995) 267-385, [hep-th/9405029].

[18] G. Bergner, T. Kaestner, S. Uhlmann, and A. Wipf, Annals Phys. 323 (2008) 946-988, [arXiv:0705.2212].

[19] J. Giedt, R. Koniuk, E. Poppitz, and T. Yavin, JHEP 12 (2004) 033, [hep-lat/ 0410041 ].

[20] N. Prokof'ev and B. Svistunov, Phys. Rev. Lett. 87 (2001) 160601.

[21] U. Wolff, Nucl. Phys. B789 (2008) 258-276, [arXiv: 0707.2872$].$

[22] S. Catterall and E. Gregory, Phys. Lett. B487 (2000) 349-356, [hep-lat/ 0006013 ].

[23] S. Catterall, Nucl. Phys. Proc. Suppl. 129 (2004) 871-873, [hep-lat/ 0309040 ].

[24] D. Baumgartner and U. Wenger, in progress. 